\title{
Model Predictive Control with Constant Switching Frequency Using a Discrete Space Vector Modulation with Virtual State Vectors
}

\begin{abstract}
Finite States Model Predictive Control (FS-MPC) appears as a promising control technique to be applied to power converters in the industry. However, the FS-MPC presents some drawbacks as non constant switching frequency and high sampling frequency. This work proposes a FS-MPC with constant switching frequency and low sampling frequency applying a Discrete Space Vector Modulation (DSVM) technique. The real state vectors of the converter are used together with new virtual state vectors forming switching sequences each sampling period. The advantages and disadvantages of the proposed FS-MPC with the DSVM are analyzed using a two-level three-phase inverter connected to the grid as setup to introduce the proposed technique. Simulation results are presented, showing that using the proposed technique the switching frequency is fixed and the sampling frequency can be lowered without reducing the quality of the converter behavior.
\end{abstract}

\section{INTRODUCTION}

$\mathbf{S}$ INCE power converters appear the development of different applications and their associated control strategies have been a challenge in the power electronics field. Several control techniques have been applied to the power converters. Linear controllers and non linear controllers including adaptive control, repetitive control, fuzzy control and predictive control have been widely used in different applications as rectifiers, inverters, active power filters, uninterruptible power supplies and motors control [1]-[4].

Among the predictive techniques, deadbeat controllers and Model Predictive Control (MPC) have stand out due to their good properties [5]-[8]. The implementation of MPC for power converters can be difficult due to the large amount of calculations needed to solve online the optimization problem. To overcome this, two solutions has been proposed: offline calculation of the optimal solutions [9], and calculation of the optimal solution by evaluation of all possible switching states. This last solution has been called Finite States Model Predictive Control (FS-MPC), because it takes into account only the finite number of possible switching states, and can be easily implemented using standard control hardware. In the particular case of FS-MPC, several applications as rectifier [10], inverter [11], [12], uninterruptible power supply [13] and motor control [14], have shown that this type of control presents high performance without the necessity to adjust controller parameters to obtain an optimized behavior of the overall system. In despite of the good features shown, the FS-MPC has two main drawbacks that makes very difficult its use in real industrial power converters. The FS-MPC needs a high sampling frequency to achieve high performance, this entails high computational cost and therefore expensive DSP or FPGA hardware. Besides FS-MPC applications show non constant switching with a widespread spectrum of the output currents and voltages. This fact leads to the necessity of large inductor and capacitors values for the connection passive filters, increasing the weight, volume and cost of the power converter.

These drawbacks are present in other discrete controllers as Direct Power Control (DPC) in rectifiers applications and Direct Torque Control (DTC) in motor control [15], [16]. Both control techniques are based on choosing in a look up table (LUT) the best output state to minimize the errors between the command references and the actual values of instantaneous active and reactive power (DPC), or torque and flux (DTC). To enhance the performance of DTC the use of a Discrete Space Vector Modulation (DSVM) has been proposed [17][22], improving the drive performance through the use of new states with a prefixed time intervals.

In this paper this concept is extended to the FS-MPC and the use of DSVM to carry out the FS-MPC is proposed. This new technique is called MPC-DSVM. The proposed method allows to achieve the control objectives with low sampling frequency and constant switching frequency. To assess the MPC-DSVM, simulations results are presented using a conventional twolevel three-phase inverter connected to the grid. Finally the advantages and disadvantages of the proposed FS-MPC with the DSVM are analyzed.

\section{FS-MPC}

FS-MPC is based on the minimization of a defined cost function studying the predicted response of each discrete output state of a power converter. Each sampling time $\left(T_{s}\right)$, the FS-MPC evaluates all possible output states and chooses the state that minimizes a cost function as the state to be applied to the converter. FS-MPC technique presents the advantages and disadvantages summarized in Table I and Table II respectively.

Among the most valuable characteristics of FS-MPC it can be noticed that high performance controllers can be implemented straightforward. However this requires high sampling frequency, which determines the control hardware to

TABLE I

ADVANTAGES OF FS-MPC CONTROLLERS

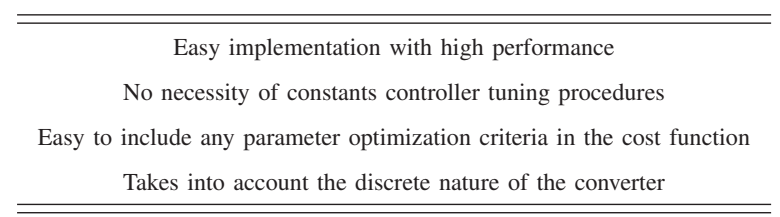


TABLE II

DRAWBACKS OF FS-MPC CONTROLLERS

Needs high sampling frequency $f_{s}$ to obtain high performance

Presents non constant switching frequency $f_{s w}$, limited by $f_{s} / 2$

Does not use the complete converter control region

be used to control the power converter. Another noticeable features are the no necessity of constants controller tuning procedures and ease to include any constrain or parameter optimization criteria in the cost function. Finally, FS-MPC takes into account the discrete nature of the converter. As a consequence, FS-MPC does not use the complete converter control region, only the discrete real states of the converter (Fig. 1). This fact together with the possibility of the cost function to choose several consecutive times the same discrete real state as the optimum state, leads FS-MPC to present non constant switching frequency $\left(f_{s w}\right)$. Besides $f_{s w}$ can be as high as half the sampling frequency $\left(f_{s}\right)$ due to there is no restriction in the switching sequence unless it is included in the cost function.

FS-MPC controllers are also characterized by the way they generate the power switch firing pulses. In comparison with external modulator based controllers, FS-MPC does not need a modulator to generate the firing pulses. The power switch gate signals can be obtained from a LUT, where the correspondence between the discrete real states of the converter and the gate signals are stored. Although it is not necessary, it is possible to generate the FS-MPC firing pulses using an external modulator as it is shown in Fig. 2. The use of an external modulator does not complicate the hardware used in the application, but opens new possibilities to FS-MPC as the complete converter control region is easily accessible.

\section{MPC-DSVM}

FS-MPC only considers the converter discrete real states to predict the system response and to evaluate the FS-MPC cost function. Although this leads to high dynamic performance of the system, the complete control region of the converter is not used and as a consequence some drawbacks appear as was shown in section II.

The idea of MPC-DSVM is to use not only the converter discrete real states but in addition other points of the control region called virtual vectors [23]. These virtual vectors can be located in any position of the converter control region and have to be synthesized by a linear combination of the converter discrete real states. Fig. 3 shows the control region of the two-level three-phase converter where the converter discrete real state vectors (round marks) and twelve additional virtual vectors (square marks) have been represented. In general, any number of virtual vectors can be plotted in the control region.

The converter virtual vectors can be generated as a linear combination of the discrete real states of the converter by using a discrete space vector modulation (DSVM). The DSVM technique is a space vector modulation based on the use of a finite number of virtual vectors placed in the control region of the power converter instead of using the whole converter

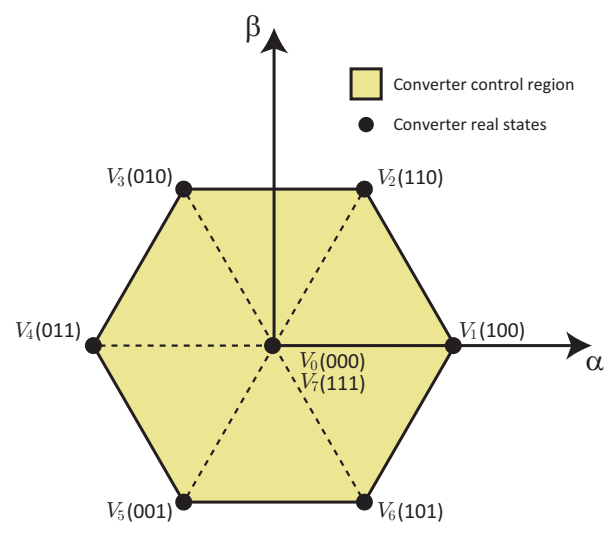

Fig. 1. Control region of a two-level three-phase converter in the $\alpha \beta$ frame.
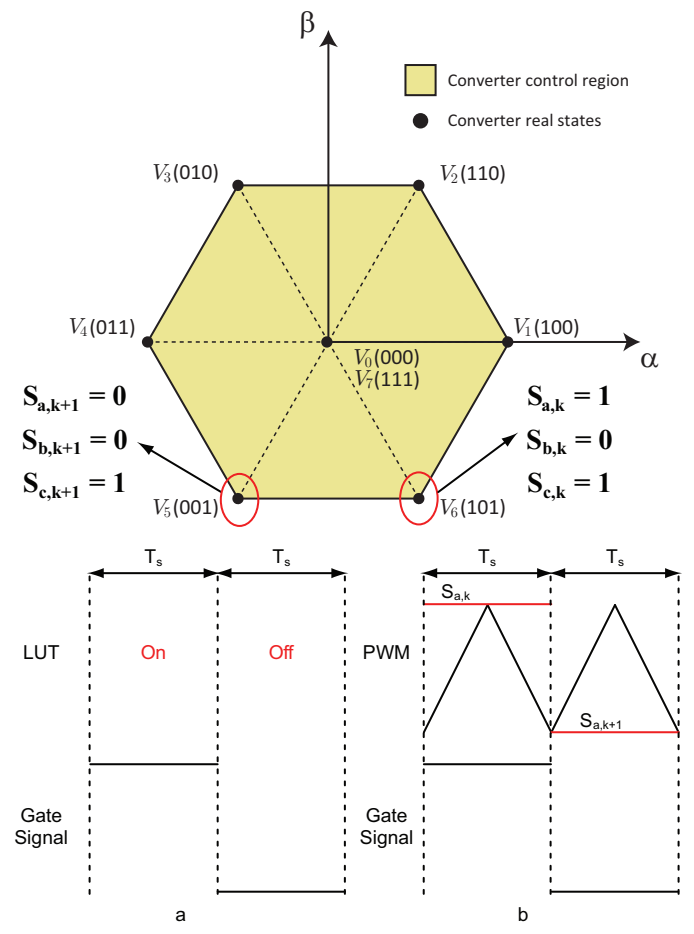

Fig. 2. Generation of the power switch firing pulses with FS-MPC. a) Through LUT. b) Through external PWM modulator.

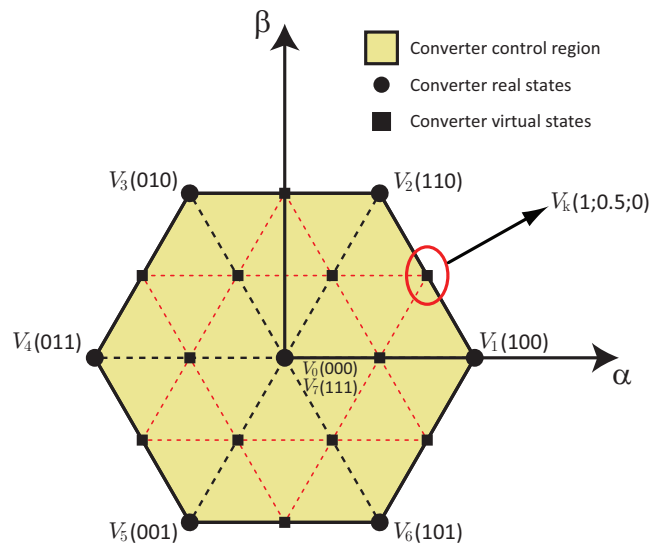

Fig. 3. Control region of a two-level three-phase converter in the $\alpha \beta$ frame including twelve virtual states. 
control region. The concept of DSVM was introduced in [17], [18], and has been used to reduce the torque and current ripple in DTC applications [19]-[22].

Any virtual vector $v^{v i r}$ can be achieved by a linear combination of three real vectors $V^{\text {real }}$, where each real vector is applied in a switching sequence a certain time quantity.

$$
\begin{gathered}
v^{v i r}=\sum_{j=1,2,3} t_{j} V_{j}^{\text {real }} \\
t_{1}+t_{2}+t_{3}=T_{s w} \\
V_{j}^{\text {real }} \in\left\{V_{0}, V_{1}, \ldots, V_{7}\right\}
\end{gathered}
$$

In (1), $T_{s w}$ represents the switching time, if $T_{s w}$ is fixed equal for all the virtual vectors then $T_{s w}$ is transformed in the switching period and the switching frequency of MPC-DSVM is fixed, solving in this way one of the mayor issues of conventional FS-MPC. As in FS-MPC, the number of vectors to be evaluated is finite so it is possible to use a LUT to store the switching vector sequence and times. However when the number of virtual vectors increases a better solution is to use an external modulator to generate the power switch gate signals as it is shown in Fig. 4.

\section{Simulations RESUlts}

MPC-DSVM technique can be employed in any power converter application that uses FS-MPC as control technique. For the shake of simplicity, to present the concept of MPC-DSVM a conventional two-level three-phase inverter connected to the grid is used. The three-phase two-level power converter is depicted in Fig. 5, where the neutral point is denoted by $n$. The system is connected to the grid through smoothing inductors $L$ and a constant DC voltage source $V_{d c}$ is connected in the DC terminals of the converter. The system parameters and variables are described in Table III.

The equations that describe the input currents dynamics can be derived from the system model in the stationary $\alpha \beta$ frame. The variables in the stationary frame are calculated as $\{\cdot\}_{\alpha \beta}^{T}=$ $A\{\cdot\}_{a b c}^{T}$.

$$
\begin{aligned}
& v_{\alpha \beta}=L \frac{d i_{\alpha \beta}}{d t}+\frac{V_{d c}}{2} S_{\alpha \beta} \\
& A=\sqrt{\frac{2}{3}}\left[\begin{array}{ccc}
1 & -\frac{1}{2} & -\frac{1}{2} \\
0 & \frac{\sqrt{3}}{2} & -\frac{\sqrt{3}}{2}
\end{array}\right]
\end{aligned}
$$

The control objective for the three-phase power inverter is the input current tracking towards their references, $i_{\alpha}^{*}$ and $i_{\beta}^{*}$

TABLE III

SYSTEM VARIABLES AND PARAMETERS

\begin{tabular}{cc}
\hline \hline Variable & Description \\
\hline$v=\left\{v_{a n} v_{b n} v_{c n}\right\}^{T}$ & Phase to neutral input voltage vector \\
$i=\left\{i_{a} i_{b} i_{c}\right\}^{T}$ & Phase input current vector \\
$S=\left\{S_{a} S_{b} S_{c}\right\}^{T}$ & Control input vector \\
$\omega$ & Grid frequency \\
$L$ & Smoothing inductor \\
$V_{d c}$ & DC voltage source value \\
\hline \hline
\end{tabular}

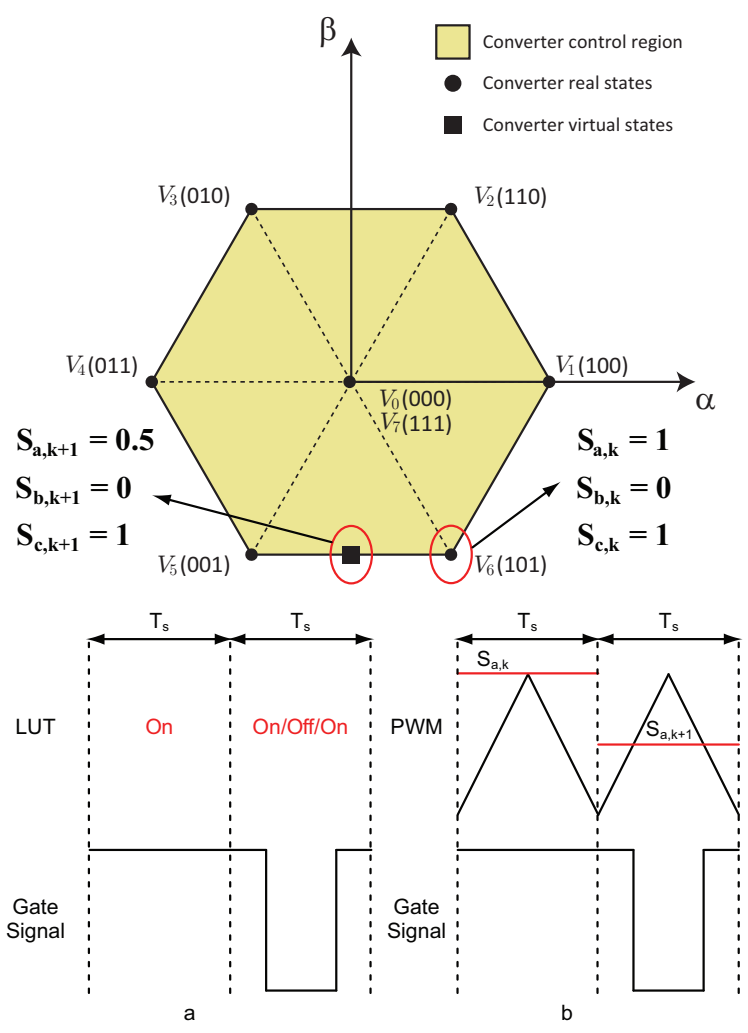

Fig. 4. Generation of the power switch firing pulses with MPC-DSVM. a) Through LUT. b) Through external PWM modulator.

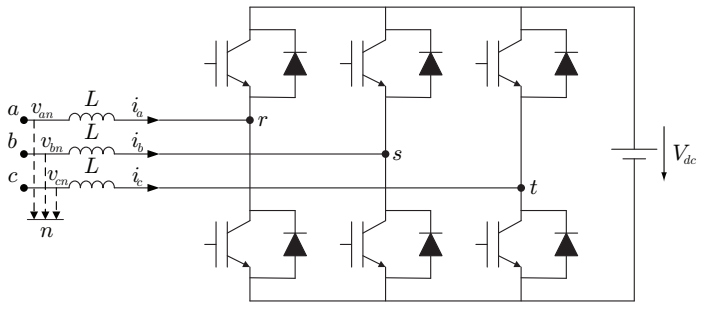

Fig. 5. Three-phase two level power inverter.

respectively. These references can be calculated in such a way that certain values of active and reactive power are injected or consumed from the grid, however this is out of scope of this paper.

$$
\begin{aligned}
& i_{\alpha} \rightarrow i_{\alpha}^{*} \\
& i_{\beta} \rightarrow i_{\beta}^{*}
\end{aligned}
$$

In [11] a FS-MPC current controller for the three-phase two-level inverter is proposed. This control algorithm has been modified as it is shown in Fig. 6, where the number of discrete output states under concern is $n_{v}$, including the evaluation of the real and virtual state vectors. The final hardware configuration is presented in Fig. 7 showing the power converter diagram block, where an external modulator has been used to generate the firing pulses.

Several simulations have been performed to analyze the influence on the power converter performance when virtual vectors are used diminishing the sampling frequency. In Table IV the parameter values used in the simulations are summarized. Four different situations have been considered. The 


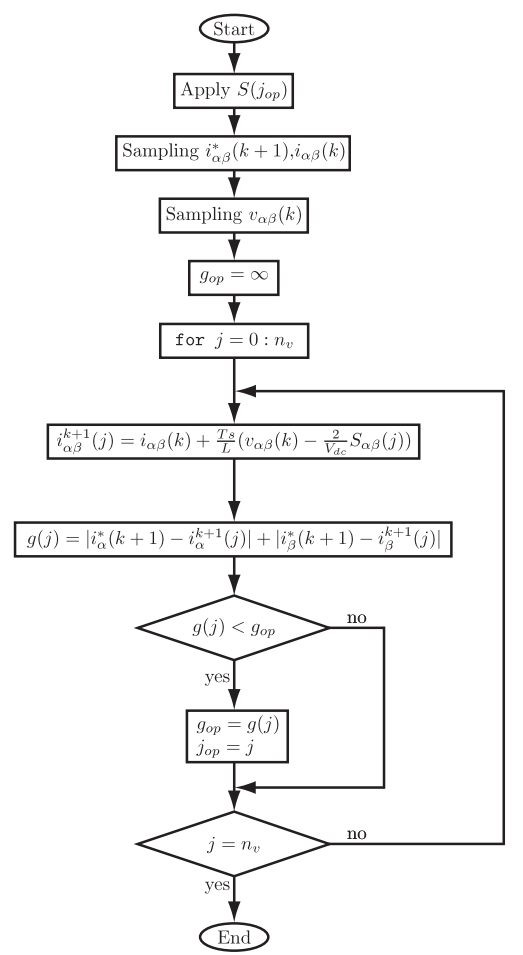

Fig. 6. Flow diagram of the MPC-DSVM control algorithm.

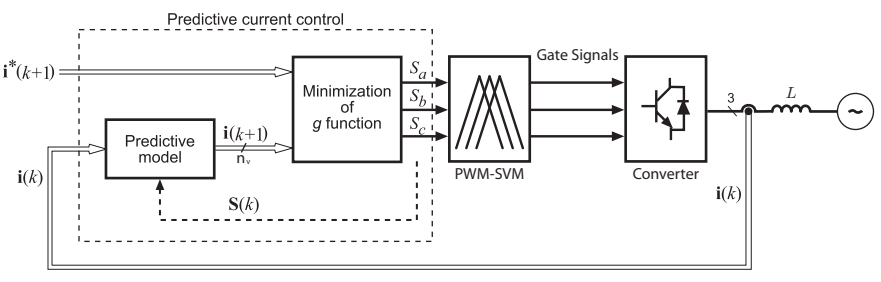

Fig. 7. Power converter diagram block using MPC-DSVM.

first one is a FS-MPC evaluating only the eight real state vectors using high sampling frequency. Secondly the FS-MPC performance is evaluated when the sampling frequency is five times slower. The third experiment uses the proposed MPC-DSVM with the same sampling frequency as the second situation, but evaluates thirty eight output vector states (eight real and thirty virtual states). In this way the computational effort has been kept constant compared with experiment 1 . Finally MPC-DSVM with a higher number of virtual vectors is considered but the sampling frequency is kept as in the second and third experiment.

In Table $\mathrm{V}$ are shown the sampling frequency and the

TABLE IV

Simulation Parameters Values

\begin{tabular}{cc}
\hline \hline Parameter & Value \\
\hline$v_{a n}, v_{b n}, v_{c n}$ & $230 \mathrm{Vrms}$ \\
$i_{a, \text { peak }}^{*}, i_{b, \text { peak }}^{*}, i_{c, \text { peak }}^{*}$ & $20 \mathrm{~A}$ \\
$\omega$ & $50 \mathrm{~Hz}$ \\
$L$ & $2 \mathrm{mH}$ \\
$V_{d c}$ & $750 \mathrm{~V}$ \\
\hline \hline
\end{tabular}

TABLE V

GRID CURRENT THD VALUES

\begin{tabular}{cccc}
\hline \hline & $f_{s}$ & $n_{v}$ & current THD \\
\hline Experiment 1 & $50 \mathrm{kHz}$ & 8 & $2.0 \%$ \\
Experiment 2 & $10 \mathrm{kHz}$ & 8 & $23.1 \%$ \\
Experiment 3 & $10 \mathrm{kHz}$ & 38 & $11.0 \%$ \\
\hline \hline
\end{tabular}

TABLE VI

GRID CURRENT THD VALUES FOR HIGH $n_{v}$

\begin{tabular}{ccccc}
\hline \hline \multicolumn{5}{c}{$f_{s}: 10 \mathrm{kHz}$} \\
\hline$n_{v}$ & 62 & 332 & 1262 & 4922 \\
current THD & $7.2 \%$ & $3.7 \%$ & $2.0 \%$ & $1.8 \%$ \\
\hline \hline
\end{tabular}

number of discrete output vectors $\left(n_{v}\right)$ used in experiments 1,2 and 3, together with the current total harmonic distortion (THD) value obtained in each case. The current THD has been calculated up to harmonic $49^{\text {th }}$. It can be noticed that experiment 1, corresponding with FS-MPC technique, presents the lowest harmonic content. Experiment 2 also corresponds with FS-MPC, but as the sampling frequency has been reduced then the performance of the controller is highly deteriorated. Finally, the proposed MPC-DSVM is performed in experiment 3 . When compared with experiment 2, the THD value has been reduced in $50 \%$, however this value is higher than THD obtained with FS-MPC in experiment 1.

Fig. 8, 9 and 10 show the grid current waveform of phase a, its reference and its harmonic spectrum for experiments 1,2 and 3 respectively. It can be noticed in Fig. 8 that current ripple for FS-MPC with high sampling frequency is very small. In addition, harmonic content is small but the spectrum is widespread. In Fig. 9 the current ripple has increased as the sampling frequency is lower, besides the spectrum is widespread as FS-MPC is used. When MPC-DSVM is employed the current ripple achieves lower values that FS-MPC with the same sampling frequency as it is shown in Fig. 10. Besides, in the harmonic spectrum can be noticed that frequency components are less spread and a peak appears in the switching frequency.

In experiment 4 is studied the performance of MPC-DSVM when the number of additional virtual vectors is increased. Table VI shows the current THD for different values of $n_{v}$. It can be noticed that THD diminishes as the number of additional virtual vector increases, being possible to obtain even lower THD than with FS-MPC. In Fig. 11 the results obtained with $n_{v}=4922$ are shown, it can be observed that current ripple is almost identical to the FS-MPC controller with high sampling frequency in experiment 1 , but in contrast having a fixed switching frequency. To study a high number of output states provides high performance to MPC-DSVM. However, MPC-DSVM has to evaluate all the discrete output vectors to minimize the cost function. The control hardware determines the maximum number of discrete output states that can be studied each sampling time, limiting in this way the performance of the system. 

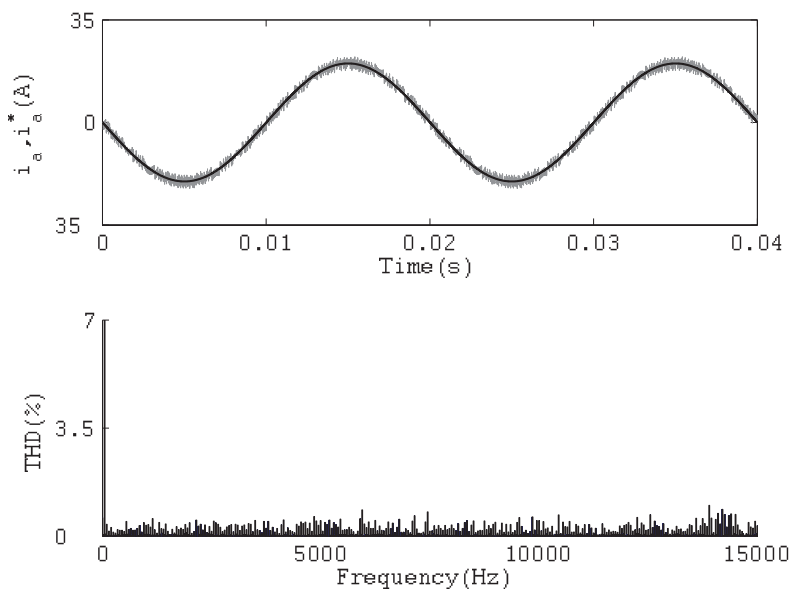

Fig. 8. Simulation results for $f_{s}=50 \mathrm{kHz}$ and $n_{v}=8$. From top to bottom: a) Phase a current and reference. b) Phase a current harmonic spectrum.
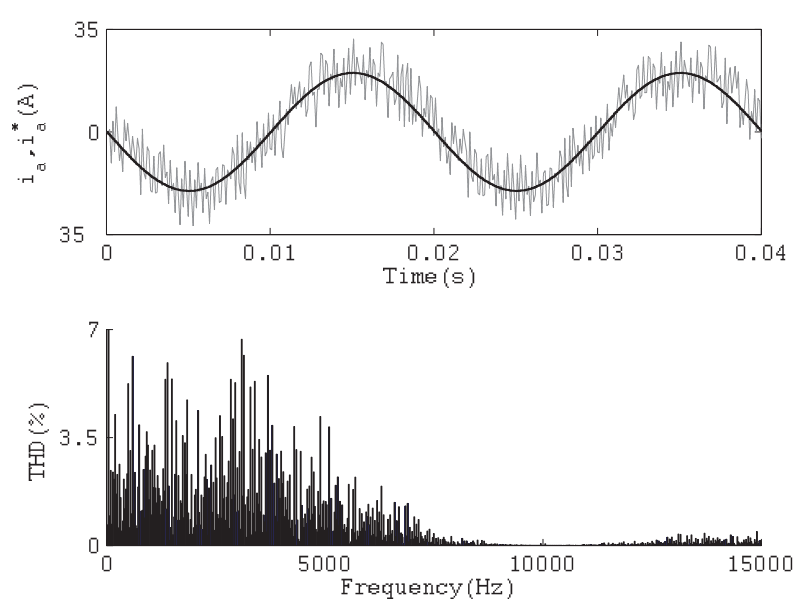

Fig. 9. Simulation results for $f_{s}=10 \mathrm{kHz}$ and $n_{v}=8$. From top to bottom: a) Phase a current and reference. b) Phase a current harmonic spectrum.

\section{ANALYSIS OF MPC-DSVM}

Tables VII and VIII summarize the advantages and disadvantages of proposed MPC-DSVM. This technique has the same advantages of FS-MPC and includes two new powerful features, constant switching frequency and low sampling frequency. Both characteristics are consequence of a better use of the converter control region. The constant switching frequency makes easier the design of passive filters and the low frequency reduces the control hardware requirements. On the other side, when MPC-DSVM is employed new concerns appear compared with FS-MPC. MPC-DSVM needs an external modulator and this forces a more complex control hardware. Modern DSPs include build-in peripherals to do this task or it can be built easily in a FPGA. Besides a new challenge emerges in low sampling frequency MPC-DSVM. To obtain similar performance to that with high sampling frequency FS-MPC it is necessary to evaluate a high number of discrete output states. This issue could be solved with a smart choosing of the virtual vectors to be analyzed in each sampling time.
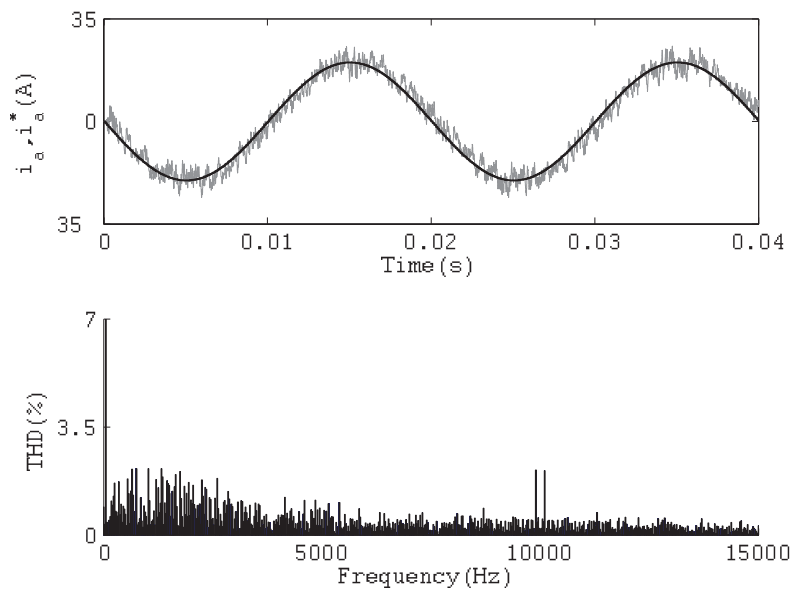

Fig. 10. Simulation results for $f_{s}=10 \mathrm{kHz}$ and $n_{v}=38$. From top to bottom: a) Phase a current and reference. b) Phase a current harmonic spectrum.
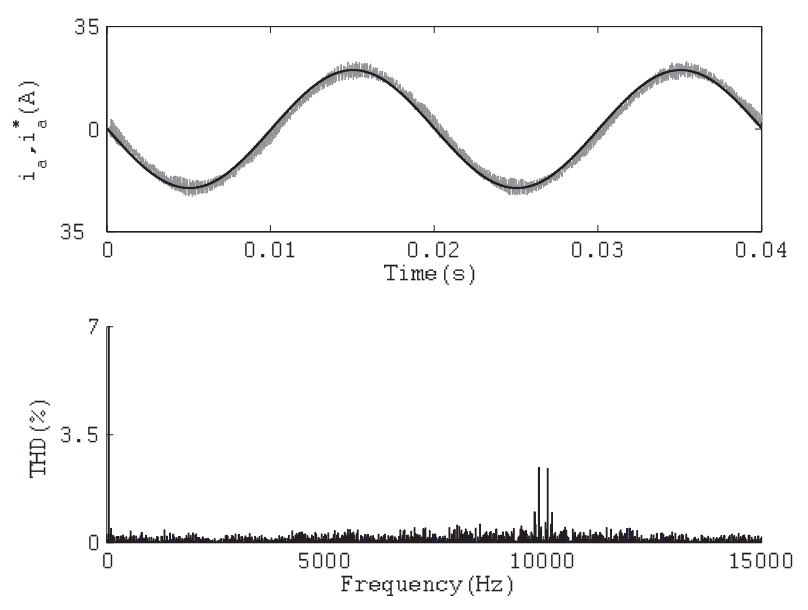

Fig. 11. Simulation results for $f_{s}=10 \mathrm{kHz}$ and $n_{v}=4922$. From top to bottom: a) Phase a current and reference. b) Phase a current harmonic spectrum.

TABLE VII

AdVANTAGES OF MPC-DSVM CONTROLLERS

Easy implementation with high performance

No necessity of constants controller tuning procedures

Easy to include any parameter optimization criteria in the cost function

Takes into account the discrete nature of the converter

Presents constant switching frequency $f_{s w}$

High performance with low sampling frequency $f_{s}$

Takes better advantage of the converter control region

TABLE VIII

DRAWBACKS OF MPC-DSVM CONTROLLERS

Needs a LUT or an external modulator to generate the firing pulses

Low $f_{s}$ increases the computational effort to achieve high performance

\section{CONCLUSIONS}

This paper presents a Finite States Model Predictive Control (FS-MPC) strategy with constant switching frequency. To 
achieve this feature, a Discrete Space Vector Modulation (DSVM) is added to the FS-MPC. The proposed MPC-DSVM is based on the evaluation of new output vectors in the controller algorithm. These output vectors, called virtual vectors, are not real converter state vectors and have to be synthesized through a external modulator. It has been shown that MPC-DSVM presents the same advantages of FS-MPC and additionally can work with low sampling frequency and fixed switching frequency. Besides, the influence of the number of output vectors evaluated have been analyzed. It has been shown that for low sampling frequency with higher performance, MPC-DSVM requires a large number of virtual vectors. However even better performance than FS-MPC can be achieved, so new challenges have been addressed.

\section{REFERENCES}

[1] S. Vazquez, J.A. Sanchez, J.M. Carrasco, J.I. Leon and E.Galvan "A model-based Direct Power Control for Three-Phase Power Converters," IEEE Transactions on Industrial Electronics, vol. 55, no. 4, pp. 1647 1657, April 2008.

[2] X. del Toro Garcia, A. Arias, M.G. Jayne and P.A. Witting, "Direct Torque Control of Induction Motors Utilizing Three-Level Voltage Source Inverters," IEEE Transactions on Industrial Electronics, vol. 55, no. 2, pp. 956-958, February 2008.

[3] C. LAscu, L. Asiminoaei, I. Boldea and F. Blaabjerg, "High Performance Current Controller for Slective Harmonic Compensation in Active Power Filters," IEEE Transactions on Power Electronics, vol. 22, no. 5, pp. 1826-1835, September 2007.

[4] J.M. Guerrero, L. Huang and J. Uceda, "Control of Distributed Uninterruptible Power Supply Systems," IEEE Transactions on Industrial Electronics, vol. 55, no. 8, pp. 2845-2859, August 2008.

[5] Y.A.R.I. Mohamed and E.F. El-Saadany, "Robust High Bandwidth Discrete-Time Predictive Current Control with Predictive Internal Model-A Unified Approach for Voltage-Source PWM Converters," IEEE Transactions on Power Electronics, vol. 23, no. 1, pp. 126-136, January 2008.

[6] E.F. Camacho and C. Bordons, Model Predictive Control. New York, N.Y.: Springer-Verlag, 1999.

[7] J.M. Maciejowski, Predictive Control with Constrains. Englewood Cliffs, N.J.: Prentice-Hall, 2002

[8] S.J. Qin and T. A. Badgwell "A survey of industrial model predictive control technology," Control Engineering Practice, vol. 11, pp. 733-764, 2003

[9] A. Linder and R. Kennel, "Model Predictive Control for Electrical Drives,' IEEE Power Electronics Specialist Conference (PESC'05), pp. 1793-1799, Recife, Brazil, 12-16 May 2005.

[10] P. Antoniewicz and M. P. Kazmierkowski, "Predictive direct power control of three-phase boost rectifier," Bulletin of Polish Academy of Science, vol. 54, no. 3, pp. 287-292,2006.
[11] J. Rodriguez, J. Pontt, C.A. Silva, P. Correa, P. Lezana, P. Cortes and U. Ammann, "Predictive Current Control of a Voltage Source Inverter," IEEE Transactions on Industrial Electronics, vol. 54, no. 1, pp. 495-503, February 2007.

[12] P. Cortes, J. Rodriguez, D.E. Quevedo and C.A. Silva, "Predictive "Predictive Current Control Strategy With Imposed Load Current Spectrum," IEEE Transactions on Power Electronics, vol. 23, no. 2, pp. 612-618, March 2008.

[13] P. Cortes, J. Rodriguez, "Three-phase inverter with output LC filter using predictive control for UPS applications," in European Conference on Electronics and Applications, Aalborg, Denmark, 2-5 September 2007

[14] J. Rodriguez, J. Pontt, C. Silva, P. Cortes, S. Rees, and U. Ammann, "Predictive direct torque control of an induction machine," in 11th International Power Electronics and Motion Control Conference, EPEPEMC 2004, Riga, Latvia, 2-4 September 2004.

[15] T. Noguchi, H. Tomiki, S. Kondo and I. Takahashi. "Direct Power Control of PWM Converter Without Power-Source Voltage Sensors," IEEE Transactions on Industry Applications, vol. 34, no. 3, pp. 473379, May/June 1998.

[16] I.Takahashi and T. Noguchi,"A New Quick-Response and HighEfficiency Control Strategy of an Induction Motor," IEEE Transactions on Industry Applications, vol. 22, no. 5, pp. 820-827, September 1986.

[17] D. Casadei, G. Serra and A. Tani, "Improvement of direct torque control performance by using a discrete SVM technique,' IEEE Power Electronics Specialist Conference (PESC'98), vol. 2, pp. 997-1003, May 1998.

[18] D. Casadei, G. Serra and A. Tani, "Implementation of a direct control algorithm for induction motors based on discrete space vector modulation," IEEE Trans. Power Electron., vol. 15, no. 4, pp. 769-777, July 2000.

[19] Junfeng Xu, Jianping Xu, Yinglei Xu and Fengyan Wang, "Direct torque control of induction machines using discrete space vector modulation applied to traction," The Fifth International Conference on Power Electronics and Drive Systems (PEDS 2003), vol. 2, pp. 1200-1202, 17-20 November 2003

[20] H. R. Keyhani, M.R. Zolghadri and A. Homaifar, "An extended and improved discrete space vector modulation direct torque control for induction motors," IEEE Power Electronics Specialists Conference (PESC'04), vol. 5, pp. 3414-3420, 20-25 June 2004.

[21] D. Ocen, L. Romeral, J. A. Ortega, J. Cusido and A. Garcia, "Discrete Space Vector Modulation Applied on a PMSM Motor," IEEE Power Electronics Specialists Conference (PESC'06), pp. 1-5, 18-22 June 2004.

[22] B. Singh and D. Goyal, "Improved DSVM-DTC Based Current Sensorless Permanent Magnet Synchronous Motor Drive," The Seventh International Conference on Power Electronics and Drive Systems (PEDS 2007), pp. 1354-1360, 27-30 Nov. 2007.

[23] J. Restrepo, J. Viola, J.M. Aller and A. Bueno, "A Simple Switch Selection State for SVM Direct Power Control," IEEE International Sysmposium on Industrial Electronics ISIE'06, pp. 1112-1116, Montreal, Canada, 9-13 July 2006. 